Provided for non-commercial research and education use. Not for reproduction, distribution or commercial use.

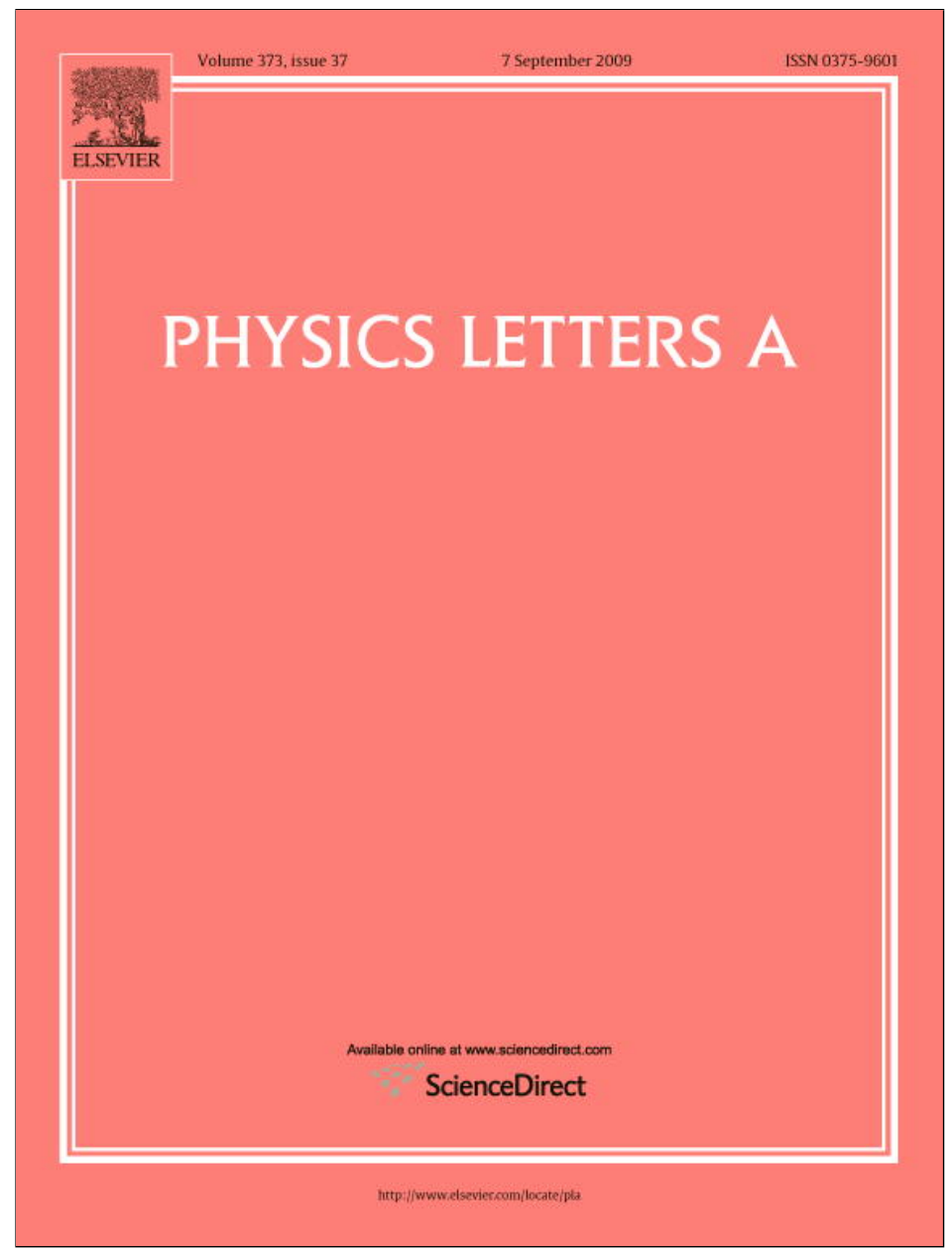

This article appeared in a journal published by Elsevier. The attached copy is furnished to the author for internal non-commercial research and education use, including for instruction at the authors institution and sharing with colleagues.

Other uses, including reproduction and distribution, or selling or licensing copies, or posting to personal, institutional or third party websites are prohibited.

In most cases authors are permitted to post their version of the article (e.g. in Word or Tex form) to their personal website or institutional repository. Authors requiring further information regarding Elsevier's archiving and manuscript policies are encouraged to visit:

http://www.elsevier.com/copyright 


\title{
Self-organization of quasi-equilibrium steady-state condensation in accumulative ion-plasma devices
}

\author{
V.I. Perekrestov $^{\text {a,* }}$, A.I. Olemskoi ${ }^{\text {a,b }}{ }^{\text {, Yu.O. Kosminska }}{ }^{\text {a }}$, A.A. Mokrenko ${ }^{\text {a }}$ \\ a Sumy State University, 2, Rimskii-Korsakov St., 40007 Sumy, Ukraine \\ b Institute of Applied Physics, 58, Petropavlovskaya St., 40030 Sumy, Ukraine
}

\section{A R T I C L E I N F O}

\section{Article history:}

Received 9 December 2008

Received in revised form 12 June 2009

Accepted 15 July 2009

Available online 21 July 2009

Communicated by A.R. Bishop

PACS:

05.65.+b

52.77.Dq

68.55.-a

81.15.Cd

81.16.-c

Keywords:

Self-organization

Stationary condensation

Supersaturation

\begin{abstract}
A B S T R A C T
We consider both theoretically and experimentally self-organization process of quasi-equilibrium steadystate condensation of sputtered substance in accumulative ion-plasma devices. It has been shown that the self-organization effect is caused by self-consistent variations of the condensate temperature and the supersaturation of depositing atoms. Two possible types of self-organization process have been found out on the basis of the phase-plane method. The aluminium condensation experimental data confirming the self-organization nature of quasi-equilibrium steady-state condensation are discussed.
\end{abstract}

(C) 2009 Elsevier B.V. All rights reserved.

\section{Introduction}

Formation of various nanosystems in the course of the condensation process is known to be achieved due to the widespread technologies, such as the molecular-beam epitaxy, the metalorganic vapour-phase epitaxy, the electrolytic deposition, the liquid-phase epitaxy, etc. [1,2]. The mentioned above technologies are characterized by two main peculiarities:

- the condensation process goes at sufficiently low supersaturation to provide a proximity to the phase equilibrium between condensate and depositing substance;

- this supersaturation remains stable in time to guarantee the steady-state conditions of the condensation process.

Due to above conditions adsorbed atoms can attach to the growth surface only if the strongest chemical bonds are realized to minimize the free energy $[3,4]$.

It is known that the proximity to phase equilibrium is determined by decrease of the supersaturation

\footnotetext{
* Corresponding author.

E-mail address: perv@phe.sumdu.edu.ua (V.I. Perekrestov).
}

$\xi=\left(n-n_{\mathrm{e}}\right) / n_{\mathrm{e}}$,

where $n$ and $n_{\mathrm{e}}$ are current and equilibrium concentration of atoms above the growth surface. In the case of vapour-condensate systems, the latter concentration is described by the following empirical relation:

$n_{\mathrm{e}}=\frac{A(T)}{k_{B} T} \exp \left(-\frac{E_{d}}{k_{B} T}\right)$,

where $E_{d}$ is the desorption energy; $T$ is the growth surface temperature; $k_{B}$ is Boltzmann constant; the temperature dependent parameter $A(T)=\exp (\alpha+\beta T+\gamma / T)$ is determined by $\alpha, \beta$ and $\gamma$, being characteristic constants for a given substance [5].

If volatile substances are used, the desorption energy is so small $\left(E_{d} \ll k_{B} T\right)$ that large value of the equilibrium concentration (2) can be expanded into series. As a result, the supersaturation becomes less dependent on the temperature change that simplifies the implementation of quasi-equilibrium steady-state condensation at relatively high deposition fluxes.

For vapour-condensate systems, e.g. using the molecular-beam epitaxy, low supersaturation is only reached due to increased growth surface temperature at relatively weak deposition fluxes. That is why the molecular-beam epitaxy can only be implemented for substances with relatively high volatility. 
The chemically active environment-condensate system, being the basis of the metal-organic vapour-phase epitaxy, the electrolytic deposition, and the liquid-phase epitaxy, the proximity to the phase equilibrium is additionally stimulated by reversible chemical processes. They induce the reverse transition of adsorbed atoms with weak bonds with the growth surface into environment. In Eqs. (1) and (2) this fact is expressed in the decrease of value $E_{d}$ of the desorption energy down to an effective value that increases the equilibrium concentration $n_{\mathrm{e}}$ and, thus, stimulates proximity to equilibrium. From this point of view, the above mentioned chemical methods make possible to reach low supersaturation at condensation of weakly volatile substances, which make the methods more universal in comparison with the molecular-beam epitaxy.

The present work is devoted to low temperature plasma-condensate system, which enables to reach a proximity to the phase equilibrium by means of heating of the growth surface with the help of plasma stream, energy and momentum transfer from plasma particles to adatoms, and their partial thermal accommodation. Due to the latter two mechanisms the desorption energy $E_{d}$ gets an effective value $[3,6,7]$

$E=E_{d}-\delta E$,

where $\delta E$ is a stochastic addition characterized by the mean value $\bar{E}$ and the dispersion $\sigma^{2} \equiv \overline{(\delta E-\bar{E})^{2}}$ (the overline means averaging over plasma particles collisions). Therefore, the low temperature plasma-condensate systems, as well as the chemical systems, allow stimulating proximity to equilibrium at weakly volatile substances deposition. However, while using plasma, there appears a problem to fix steady-state conditions during the condensation process.

So, using all mentioned above technologies raises a range of problems how to fix steady-state regime of the condensation process at vanishing supersaturation. The main point is that such crucial technological parameters as the growth surface temperature $T$ (hence, the equilibrium concentration $n_{\mathrm{e}}=n_{\mathrm{e}}(T)$ ) and the depositing flux $J$ are mutually independent and are regulated by different power sources. Also, under quasi-equilibrium conditions, the condensing flux $J_{c}$ obeys the inequality $J_{c} \ll J$. Hence, even slight relative variations $\Delta J / J$ of the depositing flux may cause considerable changes in condensation kinetics on the condition $\Delta J \sim J_{c}$. (The same effect can cause variation of the equilibrium concentration $n_{\mathrm{e}}(T)$ at slight fluctuations of the growth surface temperature $T$.) If the self-organization is absent, these problems are solved by artificially created feedback between condensation kinetics and depositing flux by means of control systems that results in considerable rise in the cost of the technology.

Therefore, the aim of this research is double: (i) to elaborate a technological device, which operation within the selforganization regime ensures extremely low steady-state supersaturations; (ii) to create a theoretical model describing the process of self-organization. To gain the mentioned above aims, the outline of the Letter is as follows. The physical principles of accumulative ion-plasma device functioning are given in the Section 2. On the basis of these conceptions, we consider the self-organization of extremely low steady-state supersaturations theoretically in Section 3. The experimental verification of the self-organization scheme is given in Section 4, and Section 5 concludes our consideration.

\section{Physical principles of accumulative ion-plasma device functioning}

The principle components of our sputtering device are the magnetron sputterer combined with the hollow cathode, which operates under the high pressure of highly refined argon $\left(P_{\mathrm{Ar}}=\right.$ 10-30 Pa) (see Fig. 1). Such construction allows to stabilize and

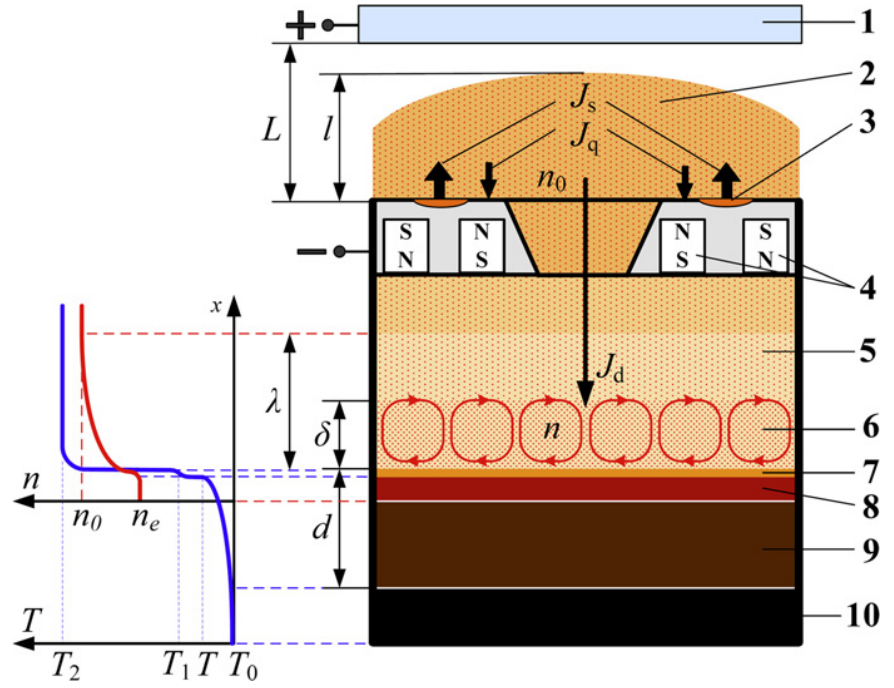

Fig. 1. (Colour online.) Scheme of axisymmetric ion-plasma device (1 - anode, 2 thermalization volume of sputtered atoms, 3 - erosion zone, 4 - magnet system, 5 - hollow cathode, 6 - area of circular mass transfer, 7 - layer of adsorbed atoms, 8 - condensate, 9 - substrate, 10 - chiller); plot on the left depicts the temperature and concentration distributions near the growth surface 7 .

increase the discharge current due to both the hollow cathode and the magnetron effects [8,9], the latter localizing the zone of erosion in addition. It is worthwhile to note that almost all atoms of inert gas and sputtered substance become ionized when get inside the hollow cathode $[8,9]$. Under the increased pressure, plasma particles intensively and frequently collide, and it makes their energy averaged. The collisions decrease the dispersion $\sigma$ of the effective desorption energy (3) that, in turn, stabilizes the condensation process.

The self-organization process starts with accumulation of sputtered substance near the growth surface. According to Ref. [10], atoms sputtered by magnetron lose their energy (i.e. thermalize) because of particles collisions in high-pressure plasma, so that their further movement becomes diffusive. Corresponding thermalization length is estimated as $l \propto\left(T_{\mathrm{Ar}}+\Delta T\right) / T_{\mathrm{Ar}} P_{\mathrm{Ar}}$, where $T_{\mathrm{Ar}}$ is the temperature of unheated working gas, and $\Delta T$ is its increment under discharge. Therefore, the thermalization length is reduced with the pressure $P_{\text {Ar }}$ increase and the discharge power decrease that reduces the temperature growth $\Delta T$. As estimations show [9, 10], the flux of non-thermalized atoms in the direction of the anode can be neglected at the pressure $P_{\mathrm{Ar}}=20-40 \mathrm{~Pa}$, the discharge power less than $100 \mathrm{~W}$, when the thermalization length is $l \leqslant L / 4$, where $L$ is the distance between the target and the anode (Fig. 1).

According to Fig. 1, the flux $J_{s}$ of sputtered substance condenses mainly on the surface adjacent to the erosion zone in the form of the flux $J_{q}$ and partially diffuses into the hollow cathode (the flux $J_{d}$ ). (It should be noted that at the initial time of the device operation, there is only inert gas plasma inside the hollow cathode.) With power up the concentration of sputtered atoms $n_{0}$ at the hollow cathode entry is determined by the fluxes $J_{e}$ and $J_{P}$, the first is drift flux caused by the presence of electric field near the inlet, and the second is caused by the pressure difference between interior and entry of the hollow cathode. As a result, the variation of the atom concentration $n_{0}$ at the hollow cathode inlet is determined by the equation

$\dot{n}_{0} \propto\left(J_{s}-J_{d}-J_{q}\right)-\left(J_{e}-J_{P}\right)$.

Here and hereinafter the point above a symbol denotes the time derivative. As the hollow cathode volume is rather small, the drift components $J_{e}$ and $J_{P}$ become quickly equal and then the sputtered substance penetrates into the hollow cathode due to diffu- 
sion only. As both $J_{s}$ and $J_{q}$ exceed the diffusive flux $J_{d}$, one can suppose that its variation slightly affects the concentration $n_{0}$. On the steady-state condition $J_{d}=J_{s}-J_{q}$ the diffusion process determines accumulation of thermalized atoms inside of the hollow cathode to increase their concentration over thermodynamic threshold $n_{\mathrm{e}}$ needed to start condensation of the sputtered substance on the substrate. ${ }^{1}$

To understand the decrease of steady-state supersaturation in the course of condensation process, let us assume that the growth surface has reached such a temperature, when the inequality $n \geqslant$ $n_{\mathrm{e}}$ starts to be fulfilled because of accumulation of sputtered substance. The supersaturation (1) takes a positive value, which initiates the barrier nucleation that, in its turn, increases the growth surface temperature because of the atom thermal accommodation. On the other hand, the flux of depositing atoms decreases due to the rupture of circular fluxes that, in turn, reduces the supersaturation to minimal value still providing condensation process. Further we are going to show that self-consistent variation of the surface temperature and the supersaturation is the most important factor of self-organization process ensuring extremely low steadystate supersaturation.

\section{Theoretical consideration of self-organization of steady-state quasi-equilibrium condensation}

Let us start with consideration of the main reasons of the surface temperature variation in the course of the ion-plasma device operation. First, each of plasma ions transfers the average energy $k_{B}\left(T_{2}-T_{1}\right)$ to the growth surface, where $T_{2}$ is the temperature of plasma ions and $T_{1}$ is the temperature of adsorbed atoms (see the temperature distribution depicted in the left panel of Fig. 1). Then, the energy delivered per time unit to the unit of the growth surface from plasma can be written in the form

$\dot{E}_{1}=\chi \theta\left(T_{2}-T\right)$,

where $\chi$ is a parameter, which is the product of Boltzmann constant and the plasma flux falling down on the growth surface, $\theta \equiv\left(T_{2}-T_{1}\right) /\left(T_{2}-T\right) \simeq 1-T_{1} / T_{2}=$ const is the thermal accommodation coefficient of adsorbed atoms rewritten for the condition $T \ll T_{2}$.

Secondly, the rate of energy transfer to the growth surface due to the thermal accommodation of condensed atoms can be shown as:

$\dot{E}_{2}=k_{B}\left(T_{2}-T\right) J_{c}$,

i.e., $\dot{E}_{2}$ is determined by the temperature difference of plasma and condensate, and also by the condensing flux $J_{c}$ :

$J_{c}=\frac{\delta}{\tau}\left(n-n_{\mathrm{e}}\right)$.

Here, $\delta$ and $\tau$ are characteristic length and time of the circle motion of condensing atoms near the growth surface. Finally, the energy removal to the chiller per time unit by means of the thermal conductivity is determined by the obvious expression

$\dot{E}_{3} \simeq \frac{\eta}{d}\left(T_{0}-T\right)$,

where $T_{0}$ stands for the chiller temperature, $d$ and $\eta$ are the total thickness and effective thermal conductivity of two-layer system consisting of condensate and substrate.

\footnotetext{
1 It is necessary to mention that under the plasma influence, the main part of deposited atoms are re-evapourated to be later ionized again and returned onto this surface under the influence of electric field [8]. Such circular mechanism of mass transfer additionally accumulates depositing atoms in immediate vicinity of the growth surface.
}

From the following obvious definition of the surface temperature variation rate

$c \dot{T}=\dot{E}_{1}+\dot{E}_{2}+\dot{E}_{3}$

combined with Eqs. (5)-(8), we obtain the differential equation

$$
\begin{aligned}
c \dot{T}= & {\left[\left(\chi \theta T_{2}+\frac{\eta}{d} T_{0}\right)+\frac{k_{B} n_{\mathrm{e}} \delta}{\tau} T_{2} \xi\right] } \\
& -\left[\left(\chi \theta+\frac{\eta}{d}\right)+\frac{k_{B} n_{\mathrm{e}} \delta}{\tau} \xi\right] T,
\end{aligned}
$$

where $c$ is the growth surface heat capacity. Eq. (10) shows the time dependence of the surface temperature which is determined by the following parameters: plasma parameters $\chi, \delta$ and $\tau$; condensate-substrate parameters $\eta, c$ and $d$; temperatures of condensate $(T)$, of plasma $\left(T_{1}, T_{2}, \theta \simeq 1-T_{1} / T_{2}\right)$, of chiller $\left(T_{0}\right)$; equilibrium concentration $n_{\mathrm{e}}$ defined by Eq. (2); and finally, supersaturation $\xi$ given by Eq. (1). It should be mentioned, that both square brackets in Eq. (10) depend on the temperature $T$ only through the equilibrium concentration (2), whereas the last term in right-hand part contains the factor $T$ itself. Moreover, the last terms in both square brackets are proportional to the supersaturation $\xi$, which time dependence should be found further.

To write down the equation for the concentration $n$, we should take into account that the condensation occurs on the inner surface of the hollow cathode with large area $S$, whereas the diffusive flux penetrates the hollow cathode through the inlet hole with small area $s$. Then the rate of concentration variation is determined by the difference between incoming diffusive and outgoing condensing fluxes, so that we obtain

$\dot{n}=\frac{1}{\delta}\left(\frac{s}{S} J_{d}-J_{c}\right)$,

where $\delta$ is the thickness of the region near the growth surface, in which accumulation of substance takes place.

According to Eq. (11), the steady-state regime is reached under the condition:

$s J_{d}=S J_{c}$,

which gives the relation $J_{c}<J_{d}$ due to the inequality $s<S$. The condensing flux is determined by Eq. (7), and the diffusive component is given by Onsager-type expression:

$J_{d} \simeq-\frac{D}{\lambda}\left(n-n_{0}\right)$,

where $\lambda$ is the effective length, where the concentration varies from $n_{0}$ to $n$, and diffusion coefficient $D$ depends on the temperature $D \sim T_{2}^{3 / 2}$ [10]. Taking into account Eqs. (2), (7), (11) and (13), the final form of the motion equation for the supersaturation (1) is as follows:

$\dot{\xi}+B(T)(1+\xi) \dot{T}=\frac{S}{S} \frac{D}{\lambda \delta} \xi_{0}-\left(\frac{1}{\tau}+\frac{S}{S} \frac{D}{\lambda \delta}\right) \xi$,

where $B(T)=\beta-\frac{1}{T}+\frac{\left(E_{d} / k_{B}\right)-\gamma}{T^{2}}$, and magnitude $\xi_{0}=\left(n_{0}-n_{\mathrm{e}}\right) / n_{\mathrm{e}}$ characterizes supersaturation at the inlet hole of the hollow cathode. Similarly to the differential equation (10), the first term in right-hand part of Eq. (14) depends on the temperature only through the equilibrium concentration (2), whereas the second term is proportional to the supersaturation, taking into account the constant factor inside the parentheses.

The system of the ordinary differential equations (10) and (14) describes self-consistent variations of the surface temperature $T$ 

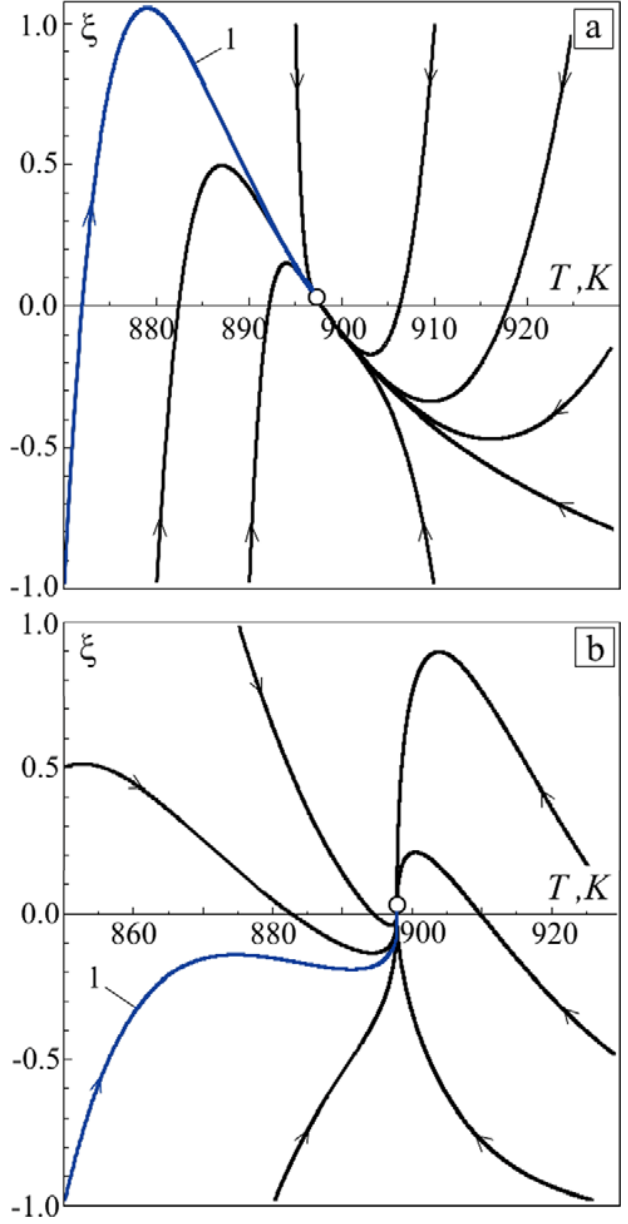

Fig. 2. (Colour online.) Phase portraits presenting solutions of Eqs. (10) and (14) at $s / S=1 / 4$ (a) and $s / S=1 / 10$ (b) (the rest of parameters is as follows: $D=$ $10^{5} \mathrm{~cm}^{2} \mathrm{~s}^{-1}, E=2.674 \times 10^{-20} \mathrm{~J}, d=0.1 \mathrm{~cm}, \lambda=1 \mathrm{~cm}, \delta=0.1 \mathrm{~cm}, \tau=10^{-6} \mathrm{~s}$, $n_{0}=10^{8} \mathrm{~cm}^{-3}, \theta=0.95, \chi=7.4 \times 10^{-3} \mathrm{JK}^{-1} \mathrm{~cm}^{-2} \mathrm{~s}^{-1}, T_{2}=6000 \mathrm{~K}, T_{0}=300 \mathrm{~K}$, $c=3 \times 10^{-3} \mathrm{~J} \mathrm{~cm}^{-2} \mathrm{~K}^{-1}, \eta=6 \times 10^{-3} \mathrm{~W} \mathrm{~cm}^{-1} \mathrm{~K}^{-1}$ (for glass). The node corresponds to $T=898 \mathrm{~K}, \xi=1.1 \times 10^{-4}, J_{c}=1.1 \times 10^{8} \mathrm{~cm}^{-2} \mathrm{~s}^{-1}$ (a) and $T=898 \mathrm{~K}, \xi=$ $7.4 \times 10^{-5}, J_{c}=7.4 \times 10^{7} \mathrm{~cm}^{-2} \mathrm{~s}^{-1}$ (b).

and the supersaturation $\xi$ during the self-organization process of extremely low steady-state supersaturations. The possible solutions of these equations are seen on the phase portraits in Fig. 2, where the set of parameters is selected for aluminium. Analysis of the phase portraits brings to the conclusion that the regime of selforganization is specifically governed by the ratio $s / S$. In the case of high values $s / S$ (Fig. 2(a)), the phase path 1 goes through high supersaturations as the temperature increases, whereas in the opposite case (Fig. 2(b)) the negative values of $\xi$ precede the steady state, corresponding to the node. From the physical point of view, in the first case, such behaviour is explained by accumulation of the sputtered substance, which advances heating of the growth surface, but in the second case, the low accumulation rate enables positive supersaturation $\xi$ only near the steady state. From the technological point of view, the second regime is much more preferable in case of little deposition time.

Under the steady-state conditions $\dot{T}=\dot{\xi}=0$, the system of Eqs. (10) and (14) shows that the steady-state supersaturation increases monotonically with the increase of effective desorption energy $E$, so that extremely low supersaturations $\xi \ll 1$ are achieved only when $E<0.2 \mathrm{eV}$. Usually, the bare desorption energy for metals is estimated as $E_{d} \sim 0.4 \mathrm{eV}$ [5], and the proximity to the phase equilibrium is provided by the plasma influence that reduces the bare value $E_{d}$ to the effective energy $E$ in accordance with Eq. (3).

\section{Experimental validation of self-organization}

We have considered the self-organization process of quasiequilibrium steady-state condensation of sputtered substance in accumulative ion-plasma devices. At the same time, numerical simulations [11-14] and experimental investigations [3,14-18] show secondary self-organization process. During such process, three-dimensional micro- and nanostructures are arranged on the substrate, possessing complicated architecture with narrow distributions over sizes and forms of structural elements. In contrast to the self-organization of the system plasma-condensate, the secondary self-organization involves in the regime of the self-organized criticality, driven by fluctuations [19]. Indeed, the primary process results in that two phase system of plasma-condensate remains near steady-state equilibrium, but strongly above a critical point, so that the phase transition is thermodynamically impossible. However, the appearance of active centres, which play the role of space distributed fluctuations, initiate the condensation process according to the following scenario. ${ }^{2}$

During the deposition process, adsorbed substance is embedded atom-by-atom into the growth surface on the active centres with the highest energies of chemical bonds. Among such centres, one can point out the mono-step kinks on the growing crystal surface, inhomogeneities on the atomic-rough surface, areas of crystal splicing and etc. [5]. Due to the reduced surface density of the active centres, the chemical bonds spectrum should be appreciably discrete. That allows distinguishing the centres, which chemical bonds energy $E_{i}, i=1,2, \ldots$ is lower than a critical threshold $E_{c}$, fixed by external conditions. ${ }^{3}$ In that case, the atom-by-atom formation of the condensate becomes improbable because of subcritical energy of chemical bonds $\left(E_{i}<E_{c}\right)$. According to the definition, the threshold $E_{c}$ is related to the desorption energy $E_{d}$ in the equilibrium concentration (2) at zero supersaturation (1), and the given concentration $n$ and the temperature T. According to Eqs. (1) and (2), the critical energy of chemical bonds

$E_{c}=k_{B} T \ln \left[\frac{A(T)}{n k_{B} T}\right]$

increases if tending to the phase equilibrium due to concentration decrease and the temperature growth.

In our experiment we investigated the structure of aluminium condensates, produced in proximity to equilibrium in the plasmacondensate system. The scanning electron microscopy images of the ex-situ grown condensates are shown in Fig. 3. These condensates were obtained on glass substrates by means of the accumulative ion-plasma system (described in Section 2) at the discharge power $1.8 \mathrm{~W}$, during the deposition time $t=9 \mathrm{~h}$, at the pressure of highly refined argon of $20 \mathrm{~Pa}$ and $15 \mathrm{~Pa}$. It is the argon pressure as technological parameter that controls supersaturation, or degree of proximity to equilibrium, at stable discharge power. Thus, if argon pressure decreases from 20 to $15 \mathrm{~Pa}$, plasma particle concentration decreases as logical result, and, therefore, their average energy increases. This reduces the effective desorption energy and accordingly to Eqs. (1) and (2) reduces supersaturation as well.

According to Fig. 3(a), if the pressure increases to $20 \mathrm{~Pa}$, we get weakly bound crystals, which habitus being fixed by crystallographic planes, which are akin to (210). The habitus is formed due to the conditions $E_{(531)}<E_{c}<E_{(210)}$, inherent in energies $E_{(h k l)}$ of atoms in half-crystal position on the ( $h k l)$-planes of fcc-metals [20]. It is necessary to stress that the structure depicted in Fig. 3(a) can be statistically homogeneous up to tens of micrometres in

\footnotetext{
2 The condensation mechanism is beyond the scope of the equilibrium thermodynamics, it is to be defined by both kinetics [4] and synergetics [19].

3 Energies of chemical bonds $E_{i}$ are considered as their absolute values.
} 


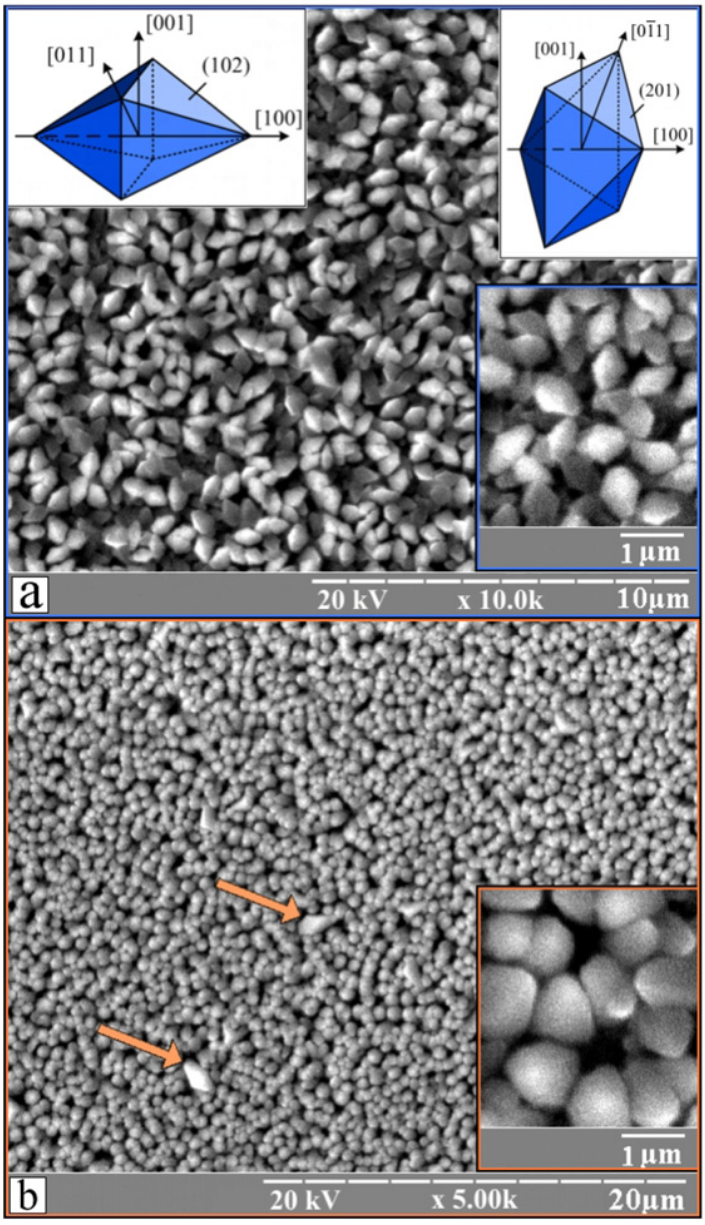

Fig. 3. (Colour online.) Scanning electron microscopy images of ex-situ grown aluminium condensates: layer-by-layer (a) and normal (b) crystal growth (the arrows point out the single crystals retaining the faceting of the previous structure). The glass substrates were used, at the argon pressure of $20 \mathrm{~Pa} \mathrm{(a)} \mathrm{and} 15 \mathrm{~Pa} \mathrm{(b),} \mathrm{the}$ discharge power $1.8 \mathrm{~W}$, the deposition time $9 \mathrm{~h}$, the area ratio $S / \mathrm{s} \sim 4$ (the condensate thickness is of order $12-14 \mu \mathrm{m}$ ).

thickness. Such formation mechanism of condensate is caused by the sequence of repeated nucleation on the active centres of the growth surface, which are usually the areas of crystallite splicing.

If the working gas pressure reduces down to $15 \mathrm{~Pa}$ and the rest of the technological parameters remain stable, the supersaturation decreases, so that the system plasma-condensate approaches to phase equilibrium. This implies that the critical energy of chemical bonds $E_{c}$ increases and becomes higher than the energy $E_{(210)}$ of atoms on the plane (210), but lower than the energy $E_{r}$ of chemical bonds on the atomic-rough surface. As a result, the condition $E_{(210)}<E_{c}<E_{r}$ is fulfilled and the mechanism of atom attaching to the growth surface is changed. The above mentioned assumption explains the transition from tangential to normal growth of round shaped crystals with atomic-rough surface (Fig. 3 ). The proximity of normal and tangential mechanisms of the crystal growth is confirmed by the fact that in Fig. 3(b) several crystals (labeled with arrows) have faceting inherent in tangential growth on the plane (210). It also should be stressed that due to quasi-equilibrium conditions of the condensation process, there is no secondary nucleation in the areas of crystallite splicing that governs the formation of columnar structures [21].

Thus, the comparison of the electron microscopy images depicted in Figs. 3(a) and 3(b) allows making a conclusion that the correspondent microstructures are obtained as result of different mechanisms of crystal growth. Obviously, above mentioned transi- tion from layer-by-layer growth to normal crystal growth is possible under two conditions: firstly, the system plasma-condensate should be extremely near the phase equilibrium (to increase the critical energy $E_{c}$ ); secondly, the condensation process should be strongly steady-state (to fix a location of the threshold $E_{c}$ in the chemical bond spectrum). As it is shown in Section 3, both conditions are provided by the self-organization of quasi-equilibrium steady-state condensation in the device presented in Section 2.

\section{Conclusion}

In contrast to the widespread technologies, we propose the original method to realize quasi-equilibrium steady-state conditions of condensation. The peculiarity of this method is atom-byatom condensation on the active centres of the growth surface, which have the strongest chemical bonds. Such regime is provided by the accumulative ion-plasma device, advantage of which in comparison with already existing systems consists in that it does not require cumbersome control system. The self-organized regime of the device operation is confirmed both theoretically and experimentally.

The above mentioned regime can be achieved by means of two principle components of the device - the magnetron sputterer and the hollow cathode. Due to diffusion, the accumulation of condensed atoms occurs inside of the hollow cathode up to the quasiequilibrium concentration, and its value remains constant during the condensation process. Heating of the growth surface with the help of plasma stream and decrease of the effective desorption energy cause extremely low supersaturation overall near the condensate.

The physical reason to reach the quasi-equilibrium steady-state condition is that condensation process evolves under scenario of the self-organization. On the basis of the phase-plane method, it is shown that self-organization is governed by the self-consistent variations of the surface temperature and the supersaturation.

To confirm the scenario of self-organization, we investigated the structure of the aluminium condensates obtained at different technological conditions. The scanning electron microscopy images showed that microstructures were formed as a result of the transforming of layer-by-layer crystal growth into normal. And this, in its turn, is possible if the plasma-condensate system is near the phase-equilibrium and the condensation process is strongly steady-state. Just these very conditions are provided by the selforganization of low steady-state supersaturation within the proposed device.

\section{Acknowledgements}

We are grateful to anonymous referees for constructive criticism.

\section{References}

[1] C. Dupas, P. Houdy, M. Lahmani, Nanoscience. Nanotechnologies and Nanophysics, Springer-Verlag, Berlin, Heidelberg, 2007.

[2] W.A. Goddart, D.W. Brenner, S.E. Lyshevsky, G.J. Iafrate (Eds.), Handbook of Nanoscience, Engineering, and Technology, CRC Press, Boca Raton, London, New York, Washington, D.C., 2003.

[3] V.I. Perekrestov, A.S. Kornyushchenko, Yu.A. Kosminskaya, JETP Lett. 86 (2007) 767.

[4] S.L. Kukushkin, A.V. Osipov, Phys. Usp. 41 (1998) 983.

[5] L.I. Maissel, R. Glang, Handbook of Thin Film Technology, McGraw-Hill, New York, 1970.

[6] F.O. Goodman, Phys. Chem. Solids 23 (1962) 1269.

[7] F.O. Goodman, Phys. Chem. Solids 24 (1963) 1451.

[8] A.G. Zhiglinskiy, V.V. Kuchinskiy, Mass Transfer at an Interaction of Plasma with Surface, Energoizdat, Moscow, 1991 (in Russian).

[9] B.S. Danylyn, Use of Low-Temperature Plasma to Deposit Thin Films, Energoatomizdat, Moscow, 1989 (in Russian). 
[10] L.G. Znamenskiy, V.L. Marchenko, Zh. Tech. Phys. 68 (1998) 24.

[11] G.K. Singh, A.A. Golovin, I.S. Aranson, Phys. Rev. B 73 (2006) 205422.

[12] F. Leroy, G. Renaud, A. Letoublon, R. Lazzari, C. Mottet, J. Goniakowski, Phys. Rev. Lett. 95 (2005) 185501.

[13] A. Ramasubramaniam, V.B. Shenoy, J. Appl. Phys. 95 (2004) 7813.

[14] P. Tejedor, P. Šmilauer, C. Roberts, B.A. Joyce, Phys. Rev. B 59 (1999) 2341.

[15] H. Omi, T. Ogino, Phys. Rev. B 59 (1999) 7521.

[16] K.A. Bratland, Y.L. Foo, J.A.N.T. Soares, et al., Phys. Rev. B 67 (2003) 125322.
[17] P. Torelli, E. Soares, G. Renand, et al., Phys. Rev. B 77 (2008) 081409.

[18] V.I. Perekrestov, A.S. Kornyushchenko, Yu.A. Kosminskaya, Phys. Solid State 50 (2008) 1357.

[19] A.I. Olemskoi, A.V. Khomenko, D.O. Kharchenko, Physica A 323 (2003) 263.

[20] B.K. Vainshtein, A.A. Chernov, L.A. Shuvalov (Eds.), Modern Crystallography III. Crystal Growth, Springer-Verlag, Berlin, 1984

[21] V.I. Perekrestov, A.S. Kornyushchenko, Yu.A. Kosminskaya, J. Tech. Phys. 53 (2008) 1364. 